both ophthalmology and rheumatology diagnoses. The same period of the year 2019 was used as a comparator.

Results: We noticed a three-fold increase in the number of ophthalmology patients who underwent an evaluation with a rheumatologist in 2020 compared to 2019 (41 vs 13, +315\%; Table). There were no differences in sex distribution or age between the two years. However, the median time before rheumatology assessment was shorter in 2020 than 2019 (median days 0 [range 0-6] vs 12 [range 0-91]). IMIDs diagnoses increased by $27 \%$ in 2020 compared to 2019 ( $29 / 41$ vs $7 / 13,71 \%$ vs $54 \%$ ), mainly driven by new diagnoses of systemic vasculitis and Behcet's disease (Figure 1). There was also an increase in diagnoses of non-IMIDs systemic conditions (3 cancers and one infection in 2020 vs none in 2019). After rheumatology evaluation, immune-modulating therapy was initiated in $18 / 41(44 \%)$ vs $3 / 13(23 \%)$.

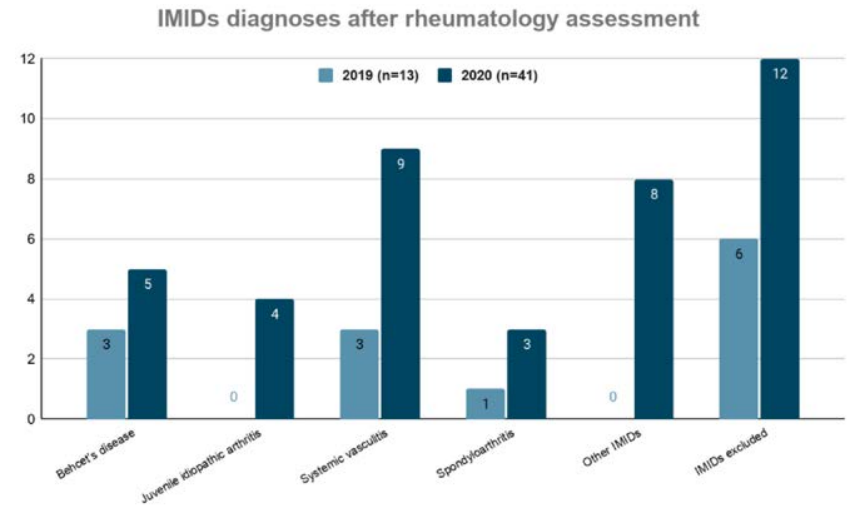

\begin{tabular}{lcc}
\hline Ophthalmology assessment and referrals to rheumatology & $\begin{array}{c}\text { Referrals } \\
\mathbf{2 0 1 9} \\
(\mathbf{n}=\mathbf{1 3})\end{array}$ & $\begin{array}{c}\text { Referrals } \\
\mathbf{2 0 2 0} \\
(\mathbf{n}=\mathbf{4 1 )}\end{array}$ \\
\hline Inflammatory eye disease, $\mathrm{n}(\%)$ & $6(46)$ & $36(88)$ \\
Uveitis, n & 6 & 21 \\
Vasculitis, $\mathrm{n}$ & 2 & 4 \\
AlON, n & 3 & 5 \\
Conjunctivitis, n & 1 & 0 \\
Scleritis, episcleritis, n & 0 & 1 \\
Other, n & 1 & 5
\end{tabular}

Conclusion: An integrated approach with rheumatologist appeared to remarkably increase the likelihood to detect IMIDs among patients presenting with inflammatory eye diseases.

Disclosure of Interests: None declared

DOI: 10.1136/annrheumdis-2021-eular.1046

\section{AB0390 CHARACTERISTICS AND PROGNOSIS OF AGE- DEPENDED ANCA-ASSOCIATED VASCULITIS IN JAPAN}

$\underline{\text { M. Kato }}^{1}$, T. Kameda ${ }^{1}$, H. Shimada ${ }^{1}$, S. Nakashima ${ }^{1}$, M. Mahmoud Fahmy Mansour ${ }^{1}$, R. Wakiya ${ }^{1}$, T. Miyagi ${ }^{1}$, K. Sugihara ${ }^{1}$, Y. Ushio ${ }^{1}$, R. Senba ${ }^{1}$, M. Mizusaki ${ }^{1}$, H. Dobashi ${ }^{1}{ }^{1}$ Kagawa University, Division of Hematology, Rheumatology and Respiratory Medicine, Department of Internal Medicine, Kagawa, Japan

Background: It is known that most of ANCA-associated vasculitis (AAV) patients are elderly. A cohort study showed that the mean age of onset was 71.1 years, especially in patients with microscopic polyangiitis (MPA) ${ }^{1)}$. However, the characteristics and prognosis of age-depended AAV patients are still unclear.

Objectives: To clarify the differences in age-related characteristics and prognosis between Japanese patients with AAV.

Methods: We enrolled 44 patients with AAV who underwent remission induction therapy at our hospital from January 2016 to December 2020. They were divided with under 70 years old group ( $<70$ yo group, $n=12$ ) and over 70 years old group ( $\geq 70$ yo group, $n=32$ ). We investigated between two groups as follows; clinical characteristics and laboratory data at diagnosis, rates of complete remission (CR) at 6 months, defined as Birmingham Vasculitis Activity
Score $($ BVAS $)=0$ and prednisone $\leq 7.5 \mathrm{mg} /$ day, adverse events, and relapse free survival.

Results: Mean age were $61.9 \pm 9.74$ years old in $<70$ yo group and $77.5 \pm 4.61$ years old in $\geq 70$ yo group $(p<0.01)$. There were significantly fewer newly diagnosed cases in the $<70$ yo group, and half of the patients with relapsing disease. There was no difference in the type of ANCA, organ involvement, or BVAS. Remission induction therapy was performed with CY or RTX, and no difference was observed between these two groups. In addition, there was also no difference in maintenance therapy (Table 1). CR rate in $<70$ yo group and in $\geq 70$ yo group were $55 \%$ and $46 \%$ respectively $(p=0.73)$. Severe infections occurred with no patient $(0 \%)$ in $<70$ yo group and with 5 patients $(16 \%)$ in $\geq 70$ yo group $(p=0.30) .5$ patients of relapse were observed in the $<70$ yo group and 1 patient in the $\geq 70$ yo group, and relapse free survival was significantly lower in the $<70$ yo group $(p=0.001)$ (Figure 1$)$.

Table 1. Clinical characteristics at baseline and treatment drugs between $<70$ yo group and $\geq 70$ yo group.

\begin{tabular}{|c|c|c|c|}
\hline & $\begin{array}{c}<70 \text { yo 群 } \\
(n=12)\end{array}$ & $\begin{array}{c}\geq 70 \text { yo 群 } \\
(n=32)\end{array}$ & $\mathrm{p}$ value \\
\hline Age (year) & $61.9 \pm 9.74$ & $77.5 \pm 4.61$ & $<0.01^{*}$ \\
\hline Female, n (\%) & $10(83)$ & $28(87)$ & 0.66 \\
\hline AAV type & $6(50) / 6(50)$ & $26(81) / 6(19)$ & 0.06 \\
\hline \multicolumn{4}{|l|}{ MPA, n (\%) / GPA, n (\%) } \\
\hline Newly diagnosed, n (\%) & $6(50)$ & $27(84)$ & $0.045^{\star}$ \\
\hline \multicolumn{4}{|l|}{ ANCA positivity } \\
\hline MPO, n (\%) / PR3, n (\%) & $11(92) / 0(0)$ & 30 (94) / 3 (9) & $1 / 0.55$ \\
\hline negative, n (\%) & $1(8)$ & $1(3)$ & 0.48 \\
\hline $\mathrm{CRP}(\mathrm{mg} / \mathrm{dl})$ & $3.34 \pm 4.01$ & $8.15 \pm 6.86$ & $0.03^{*}$ \\
\hline eGFR (mL/min) & $55.8 \pm 25.3$ & $57.7 \pm 24.7$ & 0.93 \\
\hline BVAS & $12.0 \pm 8.0$ & $14.8 \pm 6.8$ & 0.23 \\
\hline Remission induction therapy & $5(42) / 7(58)$ & $16(50) / 16(50)$ & 0.74 \\
\hline \multicolumn{4}{|l|}{ CY, n (\%) / RTX, n (\%) } \\
\hline \multicolumn{4}{|l|}{ Maintenance therapy } \\
\hline$A Z A, n(\%)$ & $7(58)$ & $14(44)$ & 0.50 \\
\hline RTX, n (\%) & $1(8)$ & $6(19)$ & 0.65 \\
\hline others, n (\%) & $2(17)$ & $3(9)$ & 0.60 \\
\hline without IS, n (\%) & $2(17)$ & $9(28)$ & 0.70 \\
\hline
\end{tabular}

The p-value was estimated using Fisher's exact or Wilcoxson rank sum test. yo, years old; AAV, ANCA-associated vasculitis; MPA, microscopic polyangiitis; GPA, granulomatosis with polyangiitis; CY, cyclophosphamide; RTX, rituximab; AZA, azathioprine; IS, immunosuppressants. ${ }^{*} \mathrm{p}<0.05$

Conclusion: There was no difference in remission rate between two groups. Severe infections were observed only in the $\geq 70$ yo group. We suggest that younger AAV patients need attention to relapse after the remission because of lower relapse free survival in $<70$ yo group.

REFERENCES:

[1] Sada KE, et al. Arthritis Res Ther 2014; 16: R101.

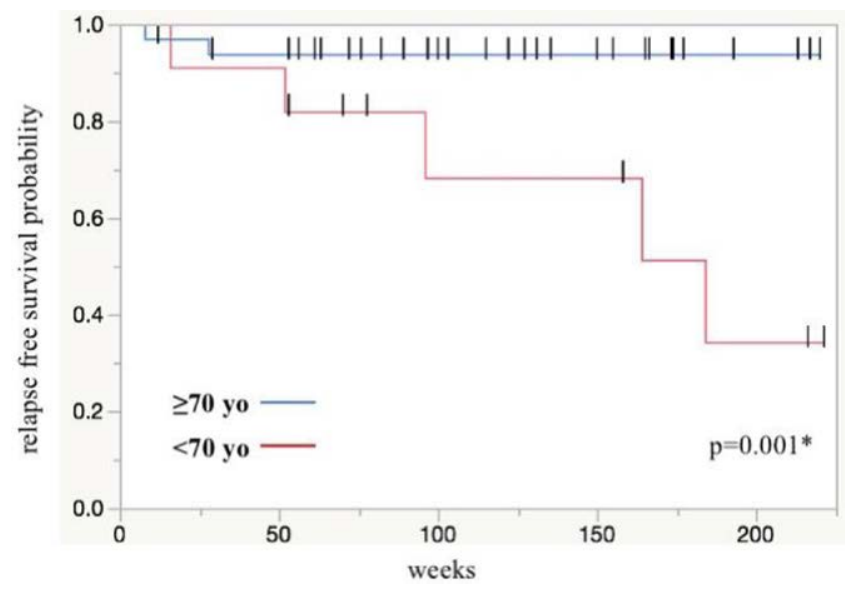

Figure 1. Relapse free survivalRelapse free survival was calculated by Kaplan-Meier method and compared by log-rank test. ${ }^{*} p<0.05$

Disclosure of Interests: None declared DOI: 10.1136/annrheumdis-2021-eular.1367 